\title{
sciendo
}

\section{Training-Induced Variations in Haematological and Biochemical Variables in Adolescent Athletes of Arab Origin Throughout an Entire Athletic Season}

\author{
by \\ Evdokia Varamenti ${ }^{1}$, Zoran Nikolovski ${ }^{1}$, Mohamed I. Elgingo ${ }^{1}$, \\ Athanasios Z. Jamurtas ${ }^{4}$, Marco Cardinale ${ }^{1,2,3}$
}

\begin{abstract}
The purpose of this study was to observe and report variations in several haematological and biochemical markers throughout an entire athletic season in a large cohort of adolescent athletes of Arab origin. Blood samples were collected from 72 adolescent male athletes at 4 selected time points during their training season. Results expressed in relation to plasma volume were corrected accordingly and significant variations in several variables emerged. Initial uncorrected haematological results revealed that haematocrit (Hct) and mean cell volume (MCV) concentrations noticeably increased at the competitive period (T3) and before the start of the following preseason (T4), whereas reticulocytes equivalent (Ret-He) only rose at T4 phase $(p<0.01)$. Conversely, corrected red blood cells (RBC), haemoglobin $(\mathrm{Hb})$ and mean cell haemoglobin concentration $(\mathrm{MCHC})$ progressively decreased over the year $(p<0.001)$. From the electrolytes panel, sodium and chloride considerably reduced at the peak of the training period (T2) to the start of the next preseason (T4), while a significant fall in potassium was mainly observed during the competitive period (T3) $(p<0.001)$. Coaches and sport scientists could use the results of this study to evaluate typical variations of each age group in order to diagnose potential adverse effects of high training loads, assist in the design of training programs and/or clinical interventions that will safeguard athletes' health, and consider the important role of plasma volume for the interpretation of results.
\end{abstract}

Key words: haematology, plasma volume, metabolites, adolescent athletes, Arab origin.

\section{Introduction}

The number of preadolescent and adolescent athletes that are involved in different sports and participate in competitions has progressively increased over the recent years culminating with the establishment of the Youth Olympic Games. To that end, there is a large number of young athletes who have been asked to follow structured and demanding programmes with training loads sometimes being comparable to those of adult athletes (Demorest and Landry, 2004).
One distinctive period in the developmental process of young people is the adolescent growth spurt or the peak height velocity (PHV) that is characterized by a rapid increase in the individual's body height and mass mainly resulting from the simultaneous increase in the secretion of testosterone and growth hormone. The pattern of human growth, in fact, is rapidly accelerated with the onset of activity within the hypothalamic-pituitary-gonadal axis

\footnotetext{
1 - Department of Sports Science, Aspire Academy, Doha (Qatar).

2 - Department of Computer Science and Institute of Sport Exercise and Health, University College London, London (UK).

3 - Faculty of Sport E Health Sciences, University of St Mark \& St John, Plymouth, (UK).

4 - Department of Physical Education and Sports Science, University of Thessaly (Greece).
} 
leading to a large increase primarily in the production of androgens (in males) and estrogen (in females). Boys experience their growth spurt about two years later, on average, than girls (i.e., boys at the age of 14 and girls at the age of 12) (Rogol, 1994). Moreover, during sports training, athletes are continuously exposed to various kinds of stress in order for adaptations to occur. As significant hormonal changes take place during puberty, any training schedule that induces hormonal and/or inflammatory effects during this critical period may have profound consequences on growth and development, especially if experienced for long periods (Eliakim and Nemet, 2013).

Screening young athletes through the year within the boundaries of what is ethically acceptable and necessary, in terms of the invasiveness of the measurements, can therefore be an appropriate and necessary approach to safeguard the athlete's health and, simultaneously, determine the implications of the applied training loads on growth and maturation. For instance, some literature reviews have concluded that athletes with suboptimal iron status may experience reduced exercise capacity and impaired sports performance (Koehler et al., 2012). Also, it has been reported that some disorders, like iron deficiency anemia, can develop as a result of rapid growth and/or the combined effect of growth and training (Hord, 1999). While the information on the variability of such measures in adolescent athletes is limited, it is recognized that some hematological variables are necessary to define aerobic fitness during growth and therefore should be periodically determined in order to assess the impact of growth and training (Winsley and Matos, 2011).

The periodic assessment of hormonal status is also relevant since the pubertal growth spurt is influenced by the release of important hormones such as steroid sex hormones that may affect the development of physical capacity and performance during childhood and adolescence (Urhaussen et al., 1995). The use of a variety of other biological markers, such as enzymes, metabolites and electrolytes, has also been proposed for assessing the implications of exercise programmes.

To our knowledge, there is a dearth of longitudinal studies in highly trained adolescent athletes assessing hematological, hormonal and enzymatic activity variability during an annual training cycle. Training can have positive or negative effects on growth, metabolites, enzymes and haematological variables depending on the training load, training specificity, age and the initial level of training. Therefore, the purpose of the study was to examine: (1) the variability in haematological markers related to the oxygen delivery system, iron status, erythropoiesis and inflammation, (2) the variability of several biochemical markers such as enzymes, metabolites, electrolytes, cortisol and sex hormone binding protein, and (3) the possible role of plasma volume shifts on examined variables throughout an annual training cycle in a group of young Arab athletes based in a sport academy in the middle east. We hypothesized that growth and maturation combined with training would induce significant improvements in some biochemical and haematological markers by the end of the annual training cycle.

\section{Methods}

\section{Participants}

Blood samples were collected from 72 adolescent male athletes of Arabic origin as part of their routine sports science and medical monitoring at a national sports academy. Measurements were carried out at 4 selected time points throughout an annual training cycle as follows: phase $T_{1}$, baseline - preseason (September); phase $T_{2}$, peak of training period (February); phase $T_{3}$, competitive period (May); and phase $T_{4}$, following year's preseason (next September). The participants were aged between 12 and 17 years and had been regularly training from 6 months to 6 years.

The mean body mass of the athletes was $55.4 \pm 15.5 \mathrm{~kg}$, with a mean body height of $164.5 \pm$ $10.6 \mathrm{~cm}$, a body mass index (BMI) of $20.2 \pm 4$ and a peak height velocity (PHV) of $0.27 \pm 1.40$ years (mean $\pm \mathrm{SD}$ ). Moreover, in order to determine seasonal changes between different age groups, the participants were divided into 3 groups based on their chronological age: group A (12-13.5 years, $N .=26)$, group $B(13.6-14.9$ years, $N .=23)$ and group C (15-16.9 years, N. = 23). Training typically involved six to nine sessions per week with varying intensity plus frequent weekly competitions. The participants practiced a wide 
variety of individual sports including athletics $(n$ $=32)$, fencing $(n=12)$, gymnastics $(n=3)$, golf $(n=$ $4)$, squash $(n=8)$, table tennis $(n=10)$, and shooting $(n=3)$. The basic criteria for taking part in this study were that the athletes had no identified injuries and were not taking medications at the time of testing.

The study was conducted in compliance with the Declaration of Helsinki and approved by the local ethics committee. Parents signed an informed consent form to allow the children to take part in the study.

\section{Anthropometric characteristics and estimation of} PHV

Anthropometric and body composition characteristics were measured one day prior to the hematological measurements. Briefly, body mass was measured with an electronic scale (Marsden, MGP250, UK); height, sitting height and leg length (Harpenden Stadiometer, Holtain, UK) were determined according to ISAK guidelines. The years from the age of peak height velocity were determined using the proposed equation (Mirwald et al., 2002). PHV estimation was included in this study instead of the Tanner scale because this method is used for athletes' growth "spurt" prediction and application of suitable training programmes.

\section{Sampling}

Participants reported to the laboratory and sat quietly for $15 \mathrm{~min}$ before providing a blood sample. Venous blood was collected each time via venipuncture from an antecubital arm vein using a safety butterfly set at rest between 7:30 and 9:30 am, after $12 \mathrm{~h}$ fasting and 2 days of minimal physical activity. The samples were collected in $3.0 \mathrm{~mL}$ K2 EDTA vacuum tubes and 4 $\mathrm{mL}$ serum separator tubes (SST 2 Advance) from Becton-Dickinson (BD, Franklin Lakes, USA). All hematological markers were evaluated on the same day in a controlled laboratory with a constant temperature of $22^{\circ} \mathrm{C}$. Serum samples were allowed to coagulate at room temperature for $30 \mathrm{~min}$ and were then centrifuged at $1500 \mathrm{~g}$ for $15 \mathrm{~min}$. After separation, samples were analyzed immediately or were stored at $-80{ }^{\circ} \mathrm{C}$ until analysed.

\section{Blood analysis}

The analysis for complete blood count and reticulocyte parameters was performed within $90 \mathrm{~min}$ of collection using a Sysmex
XT2000i hematology analyzer (Sysmex, Kobe, Japan). A certified Sysmex technician calibrated the analyzer and Sysmex e-check control standards were analyzed every day prior to sample analysis. The exercise-induced plasma volume $(\Delta \% \mathrm{PV})$ changes throughout the year were calculated accordingly (Dill and Costill, 1974). Serum samples were analysed for total iron, ferritin, aspartate aminotransferase (AST), alanine aminotransferase (ALT), creatine kinase (CK), glucose, creatinine, cholesterol, high-density lipoprotein (HDL), low-density lipoprotein (LDL) and electrolytes (sodium, potassium, chloride) on a Dimension XPand/RxL (Siemens, Erlangen, Germany). Serum samples were also assayed to determine the concentration of cortisol (C), and sex hormone binding globulin (SHBG) with ELISA kits from R\&D diagnostics (R\&D, International Inc., New York, USA). An erythrocyte sedimentation rate test (ESR) was also performed using automatic erythrocyte sedimentation analyser (Sediplus S200, SARSDEDT). The interassay coefficient of variation (CV) for cortisol ranged from 9.3 to $21.2 \%$, and for SHBG from 3.6 to $6.7 \%$. The variation coefficient (intra-assay variability) was $<5 \%$ in all the measurements.

\section{Statistical analysis}

Data are presented as age groups and as entire sample mean values and standard deviations. Data were tested for normal distribution using the Shapiro-Wilk test. Furthermore, data were examined using analysis of variance (ANOVA) with repeated measures. Whenever significant main effects for time, age and interaction were observed, Bonferroni post hoc analyses were conducted to identify statistically significant differences. A univariate one way ANOVA at each time point was used to establish the differences between groups. Effect sizes were determined by calculating Cohen's $d$ (Cohen, 1988). The magnitude of the effect size was classified as trivial $<0.2$, small $0.21-0.60$, moderate 0.61-1.20, large 1.21-1.99, and very large $>2.0$ (Cohen, 1988). The statistical package SPSS (version 17.0) was used for all analyses. The level of significance was set at $p<0.05$ for all statistical analyses.

\section{Results}

All haematological and iron related variables for the entire group of athletes and for 
each age group (mean \pm SD) separately are presented in Tables 1 and 2. Plasma volume was also quantified and for the whole group of athletes decreased by $1.4 \%$ after five months of regular training ( $\left.\mathrm{T}_{1-2}\right)$, by $4.9 \%$ before the competitions in May ( $\left.\mathrm{T}_{1-3}\right)$ and by $3.7 \%$ before the start of the next preseason. From haematological variables, haematocrit $(\mathrm{Hct})\left(\mathrm{T}_{1-3}, \mathrm{~T}_{1-4}, \mathrm{~T}_{2-3}, \mathrm{~T}_{2-4}, \mathrm{~F}=\right.$ 43.010; $p<0.001$; Cohen's $d=0.69$ ), mean corpuscular volume (MCV) $\left(\mathrm{T}_{1-3,}, \mathrm{~T}_{1-4}, \mathrm{~T}_{2-3,3}, \mathrm{~T}_{2-4}, \mathrm{~T}_{3-4}, \mathrm{~F}\right.$ $=59.810 ; p<0.001$; Cohen's $d=0.64)$, and reticulocyte equivalent (Ret-He) $\left(\mathrm{T}_{1-4}, \mathrm{~T}_{3-4}, \mathrm{~F}=3.647\right.$; $p<0.014$; Cohen's $d=0.29$ ) significantly increased, whereas mean corpuscular haemoglobin $(\mathrm{MCH})$, red blood cell distribution width (RDW-CV), reticulocyte percentage (RET\%) and immature reticulocyte fraction (IRF) marginally fluctuated over the annual training cycle.

When the initial results were accounted for plasma volume changes (results in percentages or expressed quantities were not corrected) underlined significant decreases in red blood cells (RBC) $\left(\mathrm{T}_{1-3}, \mathrm{~T}_{1-4}, \mathrm{~T}_{2-3}, \mathrm{~T}_{3-4}, \mathrm{~F}=12.109 ; p<0.001\right.$; Cohen's $d=0.49)$, haemoglobin $(\mathrm{Hb})\left(\mathrm{T}_{1-2}, \mathrm{~T}_{1-3,}, \mathrm{~T}_{1-4}\right.$, $\mathrm{T}_{2-3,3} \mathrm{~T}_{2-4}, \mathrm{~F}=3.126 ; p<0.027$; Cohen's $\left.d=0.16\right)$, and mean cell haemoglobin content $(\mathrm{MCHC})\left(\mathrm{T}_{1-2}, \mathrm{~T}_{1-3}\right.$, $\mathrm{T}_{1-4}, \mathrm{~T}_{2-3}, \mathrm{~T}_{2-4}, \mathrm{~T}_{3-4}, \mathrm{~F}=775.9 ; p<0.001$; Cohen's $d=$ 2.48) were observed throughout the annual training cycle, while ferritin concentration decreased significantly in February $(\mathrm{F}=1.467 ; p<$ 0.05 ; Cohen's $d=0.01$ ). In general, the effect size of the yearly changes ranged from trivial to small with only Hct, MCV and MCHC showing a moderate to very large effect size (Cohen's $d>$ $0.61)$.

As between groups comparisons were conducted at each testing phase, the oldest athletes (Group C) showed higher values for $\mathrm{Hb}$, Hct, MCV, MCH, WBCs and ferritin $(p<0.05)$ compared to the other two younger groups through the annual training cycle. No significant difference was identified between group $\mathrm{A}$ and group B for the same period.

All results regarding white blood cell populations for the entire group of athletes and for each age group (mean $\pm \mathrm{SD}$ ) are presented in Table 3. White blood cells (WBC) and subpopulations did vary significantly during the year (all with a trivial effect size with Cohen's $d \leq$ $0.2)$. Moreover, significant decreases over the year were noted for platelets $\left(\mathrm{T}_{1-2}, \mathrm{~T}_{1-3}, \mathrm{~T}_{1-4}, \mathrm{~F}=14.008 ; p\right.$
$<0.001$; Cohen's $d=0.427)$ and ESR $\left(\mathrm{T}_{1-2,}, \mathrm{~T}_{1-3}, \mathrm{~F}=\right.$ 7.367; $p<0.001$; Cohen's $d=0.17$ ), however, the effect size of the observed changes was small as well.

All data about the rest of the analysed variables for the entire group of athletes and for each age group (mean \pm SD) are shown in Tables 4 and 5. From the metabolites panel, creatinine $\left(\mathrm{T}_{1-2}\right.$, $\mathrm{T}_{1-3,}, \mathrm{~T}_{1-4}, \mathrm{~T}_{2-4}, \mathrm{~T}_{3-4}, \mathrm{~F}=12.586 ; p<0.001$; Cohen's $d=$ 0.42 ) increased over the examined period, lowdensity lipoprotein (LDL) increased from the start of the annual training cycle till the peak of the training period in February $\left(\mathrm{T}_{1-2}, \mathrm{~T}_{2-4}, \mathrm{~F}=3.627 ; p<\right.$ 0.014; Cohen's $d=0.08$ ), while high-density lipoprotein (HDL) reached its lowest concentration at the start of the following athletic preseason ( $\mathrm{T}_{1-4}, \mathrm{~T}_{2-4}, \mathrm{~F}=26.505 ; p<0.001$; Cohen's $d$ $=0.53$ ). Cortisol levels showed a significant reduction from the first screening to the start of the next preseason, with a moderate effect size ( $\mathrm{T}_{1}$ 4, $\mathrm{T}_{2-4}, \mathrm{~T}_{3-4}, \mathrm{~F}=17.832 ; p<0.001$; Cohen's $d=0.93$ ). $\mathrm{A}$ significant increase followed by a noteworthy decrease in SHBG was observed at $\mathrm{T}_{2}$ and $\mathrm{T}_{3}$ screenings, respectively, and this noticed reduction remained through to the next preseason $\left(\mathrm{T}_{1-3}, \mathrm{~T}_{1-4}, \mathrm{~T}_{2-3}, \mathrm{~T}_{2-4}, \mathrm{~T}_{3-4}, \mathrm{~F}=34.568 ; p<0.001\right.$; Cohen's $d$ $=0.16$ ). When results accounted for plasma volume, meaningful drops in sodium ( $\mathrm{T}_{1-2}, \mathrm{~T}_{1-3,3} \mathrm{~T}_{1-4}$, $\mathrm{T}_{2-3}, \mathrm{~T}_{2-4}, \mathrm{~T}_{3-4}, \mathrm{~F}=263.9 ; p<0.001$; Cohen's $d=0.28$ ), potassium $\left(\mathrm{T}_{1-3}, \mathrm{~T}_{1-4}, \mathrm{~T}_{2-3}, \mathrm{~T}_{2-4}, \mathrm{~F}=8.651 ; p<0.001\right.$; Cohen's $d=0.21)$ and chloride ( $\mathrm{T}_{1-2,}, \mathrm{~T}_{1-3}, \mathrm{~T}_{1-4}, \mathrm{~T}_{2-3}, \mathrm{~T}_{2-4}$, $\mathrm{T}_{3-4,} \mathrm{~F}=31.499 ; p<0.001$; Cohen's $d=1.39$ ) were observed throughout the year.

In each testing period, our results identified significant differences between the younger group $\mathrm{A}$ and the oldest in age group $\mathrm{C}$. More specific group $C$ had higher creatinine and cortisol and lower HDL and SHBG compared to group A.

\section{Discussion}

Haematological markers related to the oxygen
delivery system, iron status and erythropoiesis
Consensus on the variability in hematological variables over time among athletes and non-athletes (Joksimovic et al., 2009; Nikolaidis et al., 2003) or seasonal differences within the same squad (Andelcovic et al., 2015; Manna et al., 2010) is lacking. The current work is, to our knowledge, the first providing information about young athletes of Arab origin. 
Table 1

Haematological variables for the entire group of athletes and for each age group (mean \pm SD) at baseline, peak of the annual training cycle, competitions and at the beginning of the following preseason.

\begin{tabular}{|c|c|c|c|c|c|c|c|}
\hline & & Baseline & $\begin{array}{c}\text { Peak } \\
\text { training }\end{array}$ & Competitions & $\begin{array}{c}\text { Next } \\
\text { preseason }\end{array}$ & $\begin{array}{c}\text { Cohen's } \\
d\end{array}$ & $p$ \\
\hline Variable & Group & $\mathrm{T}_{1}$ & $\mathrm{~T}_{2}$ & $\mathrm{~T}_{3}$ & $\mathrm{~T}_{4}$ & $\mathrm{~T}_{1}-\mathrm{T}_{4}$ & \\
\hline \multirow{5}{*}{$\begin{array}{l}\text { RBC } \\
{\left[10^{6} / \mu \mathrm{l}\right]}\end{array}$} & Group A & $5.0 \pm 0.4$ & $5.1 \pm 0.4$ & $5.1 \pm 0.5$ & $5.0 \pm 0.4$ & 0 & \\
\hline & Group B & $5.0 \pm 0.5$ & $5.2 \pm 0.4$ & $5.1 \pm 0.5$ & $5.1 \pm 0.5$ & 0.19 & \\
\hline & Group C & $5.1 \pm 0.4$ & $5.1 \pm 0.4$ & $5.2 \pm 0.4$ & $5.2 \pm 0.4$ & 0 & \\
\hline & Total & $5.1 \pm 0.4$ & $5.1 \pm 0.4$ & $4.9 \pm 0.4^{*+}$ & $4.9 \pm 0.4^{*+}$ & 0.49 & \\
\hline & Corrected & $5.1 \pm 0.4$ & $5.0 \pm 0.4$ & $4.6 \pm 0.4^{*+}$ & $4.7 \pm 0.4^{*+}$ & 0.49 & $p<.001$ \\
\hline \multirow{5}{*}{$\begin{array}{l}\mathrm{Hb} \\
{[\mathrm{g} / \mathrm{dl}]}\end{array}$} & Group A & $13.1 \pm 1.3$ & $13.3 \pm 1.2$ & $13.4 \pm 1.0$ & $13.5 \pm 1.2^{*+}$ & 0.31 & \\
\hline & Group B & $13.6 \pm 1.2$ & $14.0 \pm 0.9$ & $14.0 \pm 1.0$ & $14.0 \pm 1.0$ & 0.36 & \\
\hline & Group C & $14.6 \pm 1.0$ & $14.6 \pm 1.3$ & $14.7 \pm 0.7$ & $14.6 \pm 0.8$ & 0 & \\
\hline & Total & $13.7 \pm 1.3$ & $13.9 \pm 1.2$ & $14.0 \pm 1.0^{*}$ & $14.0 \pm 1.1^{* \dagger}$ & 0.25 & \\
\hline & Corrected & $13.7 \pm 1.3$ & $13.5 \pm 1.3^{*}$ & $13.3 \pm 1.0^{*}+$ & $13.5 \pm 1.1^{*+}$ & 0.16 & $p<.027$ \\
\hline \multirow{4}{*}{ Hct [\%] } & Group A & $38.3 \pm 3.2$ & $38.9 \pm 2.7$ & $40.1 \pm 2.3^{*+}$ & $40.6 \pm 2.8^{*+\S}$ & 0.73 & \\
\hline & Group B & $39.5 \pm 3.2$ & $40.3 \pm 2.4$ & $41.7 \pm 2.9^{*+}$ & $42.0 \pm 2.9^{*+}$ & 0.81 & \\
\hline & Group C & $42.2 \pm 2.4$ & $42.2 \pm 3.0$ & $43.6 \pm 1.8^{*}+$ & $43.8 \pm 2.1^{*+}$ & 0.70 & \\
\hline & Total & $39.9 \pm 3.3$ & $40.4 \pm 3.0$ & $41.8 \pm 2.8^{*+}$ & $42.1 \pm 2.9^{*+\S}$ & 0.69 & $p<.001$ \\
\hline \multirow{4}{*}{$\begin{array}{l}\mathrm{MCV} \\
{[\mathrm{fl}]}\end{array}$} & Group A & $76.7 \pm 6.6$ & $76.2 \pm 6.6$ & $79.0 \pm 6.6^{+}$ & $81.2 \pm 5.0$ & 0.76 & \\
\hline & Group B & $78.8 \pm 5.8$ & $78.4 \pm 5.9$ & $81.2 \pm 5.9^{*+}$ & $81.9 \pm 6.2^{*}+\S$ & 0.51 & \\
\hline & Group C & $81.1 \pm 5.3$ & $81.2 \pm 6.2$ & $83.8 \pm 5.5^{*+}$ & $84.7 \pm 5.1^{*+\S}$ & 0.69 & \\
\hline & Total & $78.8 \pm 6.1$ & $78.5 \pm 6.5$ & $81.3 \pm 6.3^{*+}$ & $82.6 \pm 5.6^{*+\S}$ & 0.64 & $p<.001$ \\
\hline \multirow{4}{*}{$\begin{array}{l}\mathrm{MCH} \\
{[\mathrm{pg}]}\end{array}$} & Group A & $26.3 \pm 2.7$ & $26.1 \pm 2.7$ & $26.4 \pm 2.7^{+}$ & $26.9 \pm 2.1^{+\S}$ & 0.24 & \\
\hline & Group B & $27.0 \pm 2.4$ & $27.1 \pm 2.4$ & $27.3 \pm 2.4$ & $27.3 \pm 2.4^{+\S}$ & 0.12 & \\
\hline & Group C & $28.0 \pm 2.4$ & $28.1 \pm 2.9$ & $28.2 \pm 2.4$ & $28.3 \pm 2.2^{*}$ & 0.13 & \\
\hline & Total & $27.1 \pm 2.6$ & $27.0 \pm 2.8$ & $27.2 \pm 2.6^{+}$ & $27.5 \pm 2.3^{+\S}$ & 0.12 & $p<.07$ \\
\hline \multirow{5}{*}{$\begin{array}{l}\text { MCHC } \\
{[\mathrm{g} / \mathrm{dL}]}\end{array}$} & Group A & $34.2 \pm 0.9$ & $34.2 \pm 1.1$ & $33.4 \pm 0.9^{*}+$ & $33.1 \pm 0.8^{*+\S}$ & 1.29 & \\
\hline & Group B & $34.5 \pm 1.0$ & $34.6 \pm 0.9$ & $33.5 \pm 1.0^{*+}$ & $33.3 \pm 1.3^{*+}$ & 1.03 & \\
\hline & Group C & $34.4 \pm 1.0$ & $34.4 \pm 1.1$ & $33.5 \pm 1.0^{*+}$ & $33.2 \pm 1.0^{*+}$ & 1.19 & \\
\hline & Total & $34.4 \pm 1.0$ & $34.4 \pm 1.1$ & $33.5 \pm 1.0^{*+}$ & $33.2 \pm 0.9^{*}+\S$ & 1.26 & \\
\hline & Corrected & $34.4 \pm 1.0$ & $33.9 \pm 1.1^{*}$ & $31.8 \pm 0.9^{*}+$ & $31.9 \pm 0.9^{*}+\S$ & 2.48 & $p<.001$ \\
\hline \multirow{4}{*}{$\begin{array}{l}\text { RDW- } \\
\mathrm{CV} \text { [\%] }\end{array}$} & Group A & $13.7 \pm 1.5$ & $14.1 \pm 1.8$ & $14.0 \pm 2.1$ & $13.8 \pm 1.7$ & 0.06 & \\
\hline & Group B & $13.3 \pm 1.3$ & $13.3 \pm 1.4$ & $13.2 \pm 1.5$ & $13.2 \pm 1.3$ & 0.07 & \\
\hline & Group C & $13.4 \pm 1.6$ & $13.3 \pm 1.1$ & $13.3 \pm 2.3$ & $12.9 \pm 0.9^{*+}$ & 0.38 & \\
\hline & Total & $13.5 \pm 1.5$ & $13.6 \pm 1.5$ & $13.5 \pm 2.0$ & $13.3 \pm 1.4^{+}$ & 0.13 & $p<.114$ \\
\hline
\end{tabular}

*, Significantly different from phase $T_{1},{ }^{+}$, significantly different from phase $T_{2}$;

s, significantly different from phase $T_{3},(p<.05) . R B C=$ red blood cell; $H b=$ haemoglobin;

$H c t=$ haematocrit $; M C V=$ mean corpuscular volume; $M C H=$ mean corpuscular haemoglobin; $\mathrm{MCHC}=$ mean corpuscular haemoglobin concentration; $R D W-C V=$ red blood cell distribution width - coefficient of variance. "Results in percentages or expressed quantities were not corrected. 
Table 2

Iron related variables for the entire group of athletes and for each age group (mean $\pm S D$ ) at baseline, peak of the annual training cycle, competitions and at the beginning of the following preseason.

\begin{tabular}{|c|c|c|c|c|c|c|c|}
\hline & & Baseline & $\begin{array}{c}\text { Peak } \\
\text { training }\end{array}$ & Competitions & $\begin{array}{c}\text { Next } \\
\text { preseason }\end{array}$ & $\begin{array}{c}\text { Cohen's } \\
d\end{array}$ & $p$ \\
\hline Variable & Group & $\mathrm{T}_{1}$ & $\mathrm{~T}_{2}$ & $\mathrm{~T}_{3}$ & $\mathrm{~T}_{4}$ & $\mathrm{~T}_{1}-\mathrm{T}_{4}$ & \\
\hline \multirow{4}{*}{ Ret [\%] } & Group A & $0.98 \pm 0.31$ & $0.96 \pm 0.29$ & $0.90 \pm 0.29$ & $1.00 \pm 0.30 \S$ & 0.19 & \\
\hline & Group B & $1.15 \pm 0.40$ & $1.17 \pm 0.39$ & $1.17 \pm 0.37$ & $1.18 \pm 0.42$ & 0.07 & \\
\hline & Group C & $1.15 \pm 0.30$ & $1.10 \pm 0.30$ & $1.13 \pm 0.31$ & $1.18 \pm 0.38$ & 0.08 & \\
\hline & Total & $1.09 \pm 0.35$ & $1.07 \pm 0.34$ & $1.06 \pm 0.33$ & $1.13 \pm 0.36^{\dagger \S}$ & 0.08 & $p<.418$ \\
\hline \multirow{4}{*}{$\begin{array}{l}\text { Ret-He } \\
{[p g]}\end{array}$} & Group A & $30.8 \pm 4.1$ & $31.0 \pm 3.8$ & $31.1 \pm 3.2$ & $31.9 \pm 3.0 \S$ & 0.30 & \\
\hline & Group B & $32.6 \pm 3.1$ & $32.6 \pm 3.1$ & $32.6 \pm 3.0$ & $32.1 \pm 2.9^{*}$ & 0.46 & \\
\hline & Group C & $33.2 \pm 3.2$ & $33.1 \pm 4.2$ & $33.4 \pm 3.0$ & $33.7 \pm 3.1$ & 0.15 & \\
\hline & Total & $31.4 \pm 4.8$ & $32.2 \pm 3.8$ & $32.3 \pm 3.0$ & $32.6 \pm 3.1^{*} \S$ & 0.29 & $p<.014$ \\
\hline \multirow{4}{*}{ IRF [\%] } & Group A & $6.0 \pm 3.3$ & $7.3 \pm 3.1$ & $6.2 \pm 2.4$ & $6.5 \pm 3.4$ & 0.14 & \\
\hline & Group B & $5.9 \pm 2.7$ & $6.7 \pm 2.8$ & $6.2 \pm 2.3$ & $6.0 \pm 3.1$ & 0.03 & \\
\hline & Group C & $6.3 \pm 3.0$ & $7.0 \pm 4.0$ & $7.3 \pm 2.4$ & $7.3 \pm 3.4$ & 0.31 & \\
\hline & Total & $6.2 \pm 2.9$ & $7.0 \pm 3.3^{*}$ & $6.5 \pm 2.4$ & $6.6 \pm 3.3$ & 0.15 & $p<.161$ \\
\hline \multirow{5}{*}{$\begin{array}{l}\text { Total } \\
\text { Iron } \\
{[\text { umol/L] }}\end{array}$} & Group A & $14.5 \pm 5.8$ & $14.2 \pm 7.5$ & $15.1 \pm 9.1$ & $16.7 \pm 9.8$ & 0.27 & \\
\hline & Group B & $14.5 \pm 5.3$ & $13.8 \pm 4.5$ & $16.4 \pm 5.4$ & $15.9 \pm 5.2$ & 0.26 & \\
\hline & Group C & $17.5 \pm 7.0$ & $20.2 \pm 7.7$ & $19.6 \pm 8.1$ & $17.3 \pm 8.4$ & 0.03 & \\
\hline & Total & $15.5 \pm 6.2$ & $16.0 \pm 7.3$ & $17.0 \pm 7.9$ & $16.6 \pm 8.0$ & 0.15 & \\
\hline & Corrected & $15.5 \pm 6.2$ & $15.8 \pm 7.2$ & $16.2 \pm 7.5$ & $16.0 \pm 7.7$ & 0.07 & $p<.270$ \\
\hline \multirow{5}{*}{$\begin{array}{l}\text { Ferritin } \\
{[\mathrm{ug} / \mathrm{L}]}\end{array}$} & Group A & $34.1 \pm 33.3$ & $30.5 \pm 23.1$ & $35.5 \pm 23.8$ & $28.8 \pm 18.3$ & 0.19 & \\
\hline & Group B & $31.5 \pm 14.5$ & $28.2 \pm 11.9$ & $33.9 \pm 15.0^{+}$ & $31.5 \pm 12.5^{+}$ & 0 & \\
\hline & Group C & $46.3 \pm 32.8$ & $44.4 \pm 29.3$ & $52.2 \pm 26.7$ & $55.5 \pm 42.0$ & 0.24 & \\
\hline & Total & $37.4 \pm 28.7$ & $34.3 \pm 23.5$ & $40.3 \pm 23.6^{+}$ & $38.5 \pm 29.6^{\dagger}$ & 0.03 & \\
\hline & Corrected & $37.4 \pm 28.7$ & $33.8 \pm 23.2^{*}$ & $38.3 \pm 23.4^{+}$ & $37.1 \pm 28.5$ & 0.01 & $p<.05$ \\
\hline \multirow{4}{*}{$\begin{array}{l}\text { ESR } \\
(\mathrm{mm} / \mathrm{hr})\end{array}$} & Group A & $4.6 \pm 3.2$ & $3.0 \pm 1.3^{*}$ & $3.8 \pm 2.0$ & $5.0 \pm 3.0^{\dagger \S}$ & 0.12 & \\
\hline & Group B & $4.8 \pm 3.0$ & $3.3 \pm 2.7^{*}$ & $3.3 \pm 2.6^{*}$ & $3.6 \pm 2.1^{*}$ & 0.46 & \\
\hline & Group C & $3.3 \pm 3.0$ & $2.5 \pm 1.5$ & $2.5 \pm 1.2^{*}$ & $2.4 \pm 1.5$ & 0.37 & \\
\hline & Total & $4.4 \pm 3.1$ & $3.0 \pm 1.9^{*}$ & $3.3 \pm 2.0^{*}$ & $3.7 \pm 2.5$ & 0.17 & $p<.001$ \\
\hline$\% \Delta \mathrm{PV}$ & Total & - & $\begin{array}{l}-1.4 \% \\
\left(\mathrm{~T}_{1}-\mathrm{T}_{2}\right)\end{array}$ & $\begin{array}{c}-4.9 \% \\
\left(\mathrm{~T}_{1}-\mathrm{T}_{3}\right)\end{array}$ & $\begin{array}{l}-3.7 \% \\
\left(\mathrm{~T}_{1}-\mathrm{T}_{4}\right)\end{array}$ & & \\
\hline
\end{tabular}

", Significantly different from phase $T_{1} ;{ }^{+}$, significantly different from phase $T_{2}$;

$\S$, significantly different from phase $T_{3},(p<.05)$. Ret $\%=$ reticulocyte percentage;

Ret-He = reticulocyte haemoglobin equivalent; IRF = immature reticulocyte fraction;

$E S R=$ erythrocyte sedimentation rate; $\% \triangle P V=$ plasma volume changes.

"Results in percentages or expressed quantities were not corrected. 


\section{Table}

Leukocytes, leukocytes subpopulations and platelets counts for the entire group of athletes and for each age group (mean $\pm S D$ ) at baseline, peak of the annual training cycle, competitions and at the beginning of the following preseason.

\begin{tabular}{|c|c|c|c|c|c|c|c|}
\hline & & Baseline & $\begin{array}{c}\text { Peak } \\
\text { training }\end{array}$ & Competitions & $\begin{array}{c}\text { Next } \\
\text { preseason }\end{array}$ & $\begin{array}{c}\text { Cohen's } \\
d\end{array}$ & $p$ \\
\hline Variable & Group & $\mathrm{T}_{1}$ & $\mathrm{~T}_{2}$ & $\mathrm{~T}_{3}$ & $\mathrm{~T}_{4}$ & $\mathrm{~T}_{1}-\mathrm{T}_{4}$ & \\
\hline \multirow{5}{*}{ WBC $\left[10^{3} / \mu \mathrm{l}\right]$} & Group A & $6.4 \pm 1.4$ & $6.5 \pm 1.4$ & $6.6 \pm 2.3$ & $6.8 \pm 2.0$ & 0.23 & \\
\hline & Group B & $5.5 \pm 1.4$ & $6.0 \pm 1.3^{*}$ & $5.3 \pm 1.5^{+}$ & $5.6 \pm 1.2$ & 0.07 & \\
\hline & Group C & $5.7 \pm 1.4$ & $5.8 \pm 1.5$ & $5.3 \pm 1.2^{+}$ & $5.9 \pm 1.7 \S$ & 0.12 & \\
\hline & Total & $5.8 \pm 1.4$ & $6.0 \pm 1.4$ & $5.7 \pm 1.8$ & $6.1 \pm 1.8$ & 0.18 & \\
\hline & Corrected & $5.8 \pm 1.4$ & $5.9 \pm 1.4$ & $5.4 \pm 1.7^{*+}$ & $5.9 \pm 1.7$ & 0.06 & $p<.032$ \\
\hline \multirow{4}{*}{$\begin{array}{l}\text { Neutrophils } \\
{[\%]}\end{array}$} & Group A & $43.6 \pm 8.8$ & $44.9 \pm 10.3$ & $45.0 \pm 13.1$ & $46.5 \pm 11.1$ & 0.28 & \\
\hline & Group B & $43.2 \pm 8.9$ & $46.4 \pm 10.8$ & $42.2 \pm 9.1$ & $44.4 \pm 9.9$ & 0.12 & \\
\hline & Group C & $43.4 \pm 11.2$ & $45.3 \pm 12.1$ & $46.3 \pm 10.5$ & $45.7 \pm 11.8$ & 0.19 & \\
\hline & Total & $43.4 \pm 9.4$ & $45.3 \pm 10.9$ & $45.0 \pm 11.0$ & $45.6 \pm 10.8^{*}$ & 0.21 & $p<.181$ \\
\hline \multirow{4}{*}{$\begin{array}{l}\text { Lymphocytes } \\
{[\%]}\end{array}$} & Group A & $42.3 \pm 7.5$ & $42.2 \pm 10.0$ & $40.7 \pm 11.6$ & $40.1 \pm 10.6^{*}$ & 0.23 & \\
\hline & Group B & $42.6 \pm 9.4$ & $39.1 \pm 9.4$ & $43.6 \pm 9.0^{+}$ & $40.7 \pm 9.1 \S$ & 0.20 & \\
\hline & Group C & $42.3 \pm 11.4$ & $41.9 \pm 10.8$ & $40.7 \pm 9.9$ & $40.5 \pm 11.4$ & 0.15 & \\
\hline & Total & $42.3 \pm 9.3$ & $41.0 \pm 9.9$ & $41.6 \pm 10.2$ & $40.4 \pm 10.2^{*}$ & 0.19 & $p<.05$ \\
\hline \multirow{4}{*}{$\begin{array}{l}\text { Monocytes } \\
{[\%]}\end{array}$} & Group A & $9.1 \pm 2.0$ & $8.4 \pm 1.8$ & $8.6 \pm 1.9^{*}$ & $9.6 \pm 2.6^{*}$ & 0.21 & \\
\hline & Group B & $9.2 \pm 2.0$ & $9.6 \pm 1.8$ & $9.7 \pm 2.6$ & $10.4 \pm 2.8^{*}$ & 0.49 & \\
\hline & Group C & $10.4 \pm 3.0$ & $9.6 \pm 2.2$ & $9.4 \pm 2.0$ & $10.3 \pm 2.8$ & 0.03 & \\
\hline & Total & $9.6 \pm 2.4$ & $9.2 \pm 2.0$ & $9.2 \pm 2.2$ & $10.1 \pm 2.7^{+\S}$ & 0.19 & $p<.001$ \\
\hline \multirow{4}{*}{$\begin{array}{l}\text { Eosinophils } \\
{[\%]}\end{array}$} & Group A & $4.5 \pm 2.6$ & $4.0 \pm 2.0$ & $4.1 \pm 2.1$ & $3.4 \pm 2.0^{*}$ & 0.47 & \\
\hline & Group B & $4.6 \pm 3.2$ & $4.5 \pm 2.9$ & $4.0 \pm 2.6$ & $4.0 \pm 2.6$ & 0.20 & \\
\hline & Group C & $3.4 \pm 2.6$ & $3.4 \pm 3.2$ & $3.1 \pm 2.6$ & $3.0 \pm 1.8$ & 0.17 & \\
\hline & Total & $4.2 \pm 2.8$ & $4.0 \pm 2.8$ & $3.8 \pm 2.4$ & $3.4 \pm 2.1^{*+}$ & 0.32 & $p<.013$ \\
\hline \multirow{4}{*}{$\begin{array}{l}\text { Basophils } \\
{[\%]}\end{array}$} & Group A & $0.43 \pm 0.21$ & $0.44 \pm 0.27$ & $0.47 \pm 0.30$ & $0.43 \pm 0.27$ & 0 & \\
\hline & Group B & $0.48 \pm 0.31$ & $0.42 \pm 0.25$ & $0.44 \pm 0.23$ & $0.51 \pm 0.31$ & 0.09 & \\
\hline & Group C & $0.47 \pm 0.24$ & $0.48 \pm 0.33$ & $0.40 \pm 0.26$ & $0.50 \pm 0.36$ & 0.09 & \\
\hline & Total & $0.46 \pm 0.25$ & $0.44 \pm 0.28$ & $0.43 \pm 0.26$ & $0.48 \pm 0.31 \S$ & 0.07 & $p<.240$ \\
\hline \multirow{5}{*}{$\begin{array}{l}\text { Platelets } \\
\left(10^{9} / \mathrm{L}\right]\end{array}$} & Group A & $270.0 \pm 61$ & $261.0 \pm 51$ & $259.0 \pm 52$ & $264.1 \pm 48$ & 0.11 & \\
\hline & Group B & $287.3 \pm 48$ & $283.6 \pm 53$ & $274.7 \pm 48$ & $277.5 \pm 58^{*}$ & 0.19 & \\
\hline & Group C & $264.5 \pm 48$ & $246.4 \pm 39$ & $254.8 \pm 36$ & $247.5 \pm 33^{*+}$ & 0.41 & \\
\hline & Total & $273.8 \pm 53$ & $263.3 \pm 50$ & $262.7 \pm 46^{*}$ & $262.6 \pm 46^{*}$ & 0.37 & \\
\hline & Corrected & $273.8 \pm 53$ & $259.6 \pm 49^{*}$ & $249.8 \pm 44^{*+}$ & $252.9 \pm 44^{*+}$ & 0.42 & $p<.001$ \\
\hline \multirow{4}{*}{ MPV [fL] } & Group A & $10.8 \pm 0.9$ & $11.0 \pm 1.0$ & $10.7 \pm 0.9^{\dagger}$ & $10.6 \pm 1.0$ & 0.30 & \\
\hline & Group B & $10.4 \pm 0.7$ & $10.6 \pm 0.7$ & $10.4 \pm 0.6^{+}$ & $10.0 \pm 1.2 \S$ & 0.40 & \\
\hline & Group C & $10.2 \pm 0.8$ & $10.3 \pm 0.7$ & $10.2 \pm 0.6$ & $10.3 \pm 0.7$ & 0.13 & \\
\hline & Total & $10.5 \pm 0.8$ & $10.6 \pm 0.8$ & $10.4 \pm 0.7^{*+}$ & $10.3 \pm 1.0^{+}$ & 0.22 & $p<.038$ \\
\hline \multicolumn{8}{|c|}{$\begin{array}{l}\text { *, Significantly different from phase } T_{1} ;{ }^{+} \text {, significantly different from phase } T_{2} \text {; } \\
\S \text {, significantly different from phase } T_{3},(p<.05) . W B C=\text { white blood cells; } \\
\qquad M P V=\text { mean platelet volume. }\end{array}$} \\
\hline
\end{tabular}


Table 4

Metabolites' concentrations for the entire group of athletes and for each age group (mean $\pm S D$ ) at baseline, peak of the annual training cycle, competitions and at the beginning of the following preseason.

\begin{tabular}{|c|c|c|c|c|c|c|c|}
\hline & & Baseline & $\begin{array}{c}\text { Peak } \\
\text { training }\end{array}$ & Competitions & $\begin{array}{c}\text { Next } \\
\text { preseason }\end{array}$ & $\begin{array}{c}\text { Cohen's } \\
d\end{array}$ & $p$ \\
\hline Variable & Group & $\mathrm{T}_{1}$ & $\mathrm{~T}_{2}$ & $\mathrm{~T}_{3}$ & $\mathrm{~T}_{4}$ & $\mathrm{~T}_{1}-\mathrm{T}_{4}$ & \\
\hline \multirow{5}{*}{ CK [U/L] } & Group A & $385 \pm 656$ & $252 \pm 302$ & $333 \pm 463$ & $363 \pm 556$ & 0.03 & \\
\hline & Group B & $317 \pm 277$ & $215 \pm 111^{*}$ & $258 \pm 156$ & $266 \pm 131$ & 0.23 & \\
\hline & Group C & $559 \pm 1167$ & $318 \pm 289$ & $296 \pm 158$ & $328 \pm 328$ & 0.26 & \\
\hline & Total & $412.5 \pm 770$ & $258.7 \pm 249$ & $282.9 \pm 272$ & $308.5 \pm 368$ & 0.17 & \\
\hline & Corrected & $412.5 \pm 770$ & $255.1 \pm 245$ & $269.0 \pm 259$ & $297.1 \pm 355$ & 0.19 & $p<.073$ \\
\hline \multirow{5}{*}{$\begin{array}{l}\text { Creatinine } \\
\text { [umol/L] }\end{array}$} & Group A & $55.5 \pm 11.1$ & $62.0 \pm 12.3^{*}$ & $62.7 \pm 14.2^{*}$ & $66.2 \pm 12.5^{*}$ & 0.90 & \\
\hline & Group B & $63.8 \pm 8.9$ & $66.4 \pm 12.1$ & $67.5 \pm 10.0^{*+}$ & $75.1 \pm 14.6^{*+}$ & 0.93 & \\
\hline & Group C & $75.7 \pm 10.5$ & $80.6 \pm 12.2^{*}$ & $83.5 \pm 13.1^{*+}$ & $83.8 \pm 10.9^{*}$ & 0.77 & \\
\hline & Total & $65.3 \pm 13.3$ & $69.4 \pm 14.1^{*}$ & $70.8 \pm 15.6^{*}$ & $75.0 \pm 14.5^{*} \S$ & 0.69 & \\
\hline & Corrected & $65.3 \pm 13.3$ & $68.4 \pm 19.4^{*}$ & $67.3 \pm 14.8^{*}$ & $72.2 \pm 14^{*}+\S$ & 0.42 & $p<.001$ \\
\hline \multirow{5}{*}{ ALT [U/L] } & Group A & $29.7 \pm 12.5$ & $25.0 \pm 6.0$ & $26.1 \pm 8.1$ & $25.0 \pm 6.0$ & 0.47 & \\
\hline & Group B & $28.6 \pm 13.0$ & $29.6 \pm 12.5$ & $28.5 \pm 11.1$ & $27.8 \pm 11.5$ & 0.06 & \\
\hline & Group C & $28.9 \pm 11.8$ & $27.7 \pm 7.4$ & $24.6 \pm 6.1$ & $28.7 \pm 9.2 \S$ & 0.02 & \\
\hline & Total & $28.9 \pm 11.5$ & $27.0 \pm 8.4$ & $26.0 \pm 8.2^{*}$ & $27.0 \pm 8.8$ & 0.18 & \\
\hline & Corrected & $28.9 \pm 11.5$ & $26.6 \pm 8.2$ & $24.7 \pm 7.8^{*+}$ & $26.0 \pm 8.5$ & 0.28 & $p<.002$ \\
\hline \multirow{5}{*}{ AST [U/L] } & Group A & $26.0 \pm 8.8$ & $24.4 \pm 6.4$ & $26.8 \pm 7.9$ & $25.0 \pm 7.1$ & 0.12 & \\
\hline & Group B & $24.7 \pm 6.6$ & $23.0 \pm 5.7$ & $24.3 \pm 4.7$ & $24.2 \pm 5.6$ & 0.08 & \\
\hline & Group C & $28.9 \pm 26.6$ & $22.9 \pm 7.3$ & $23.0 \pm 7.1$ & $24.8 \pm 7.9$ & 0.20 & \\
\hline & Total & $26.4 \pm 15.7$ & $23.4 \pm 6.7$ & $24.7 \pm 7.0$ & $24.9 \pm 7.0$ & 0.02 & \\
\hline & Corrected & $26.4 \pm 15.7$ & $23.1 \pm 6.6$ & $23.2 \pm 6.6$ & $24.0 \pm 6.7$ & 0.18 & $p<.078$ \\
\hline \multirow{5}{*}{$\begin{array}{l}\text { Glucose } \\
\text { [nmol/L] }\end{array}$} & Group A & $5.0 \pm 0.6$ & $5.2 \pm 0.7$ & $5.3 \pm 0.8^{*}$ & $5.2 \pm 0.7$ & 0.30 & \\
\hline & Group B & $5.2 \pm 0.7$ & $5.1 \pm 0.8$ & $5.3 \pm 0.7$ & $5.4 \pm 0.7$ & 0.28 & \\
\hline & Group C & $5.0 \pm 0.9$ & $5.1 \pm 1.0$ & $4.7 \pm 0.6$ & $5.1 \pm 0.7$ & 0.12 & \\
\hline & Total & $5.0 \pm 0.8$ & $5.1 \pm 0.8$ & $5.1 \pm 0.7$ & $5.2 \pm 0.7$ & 0.40 & \\
\hline & Corrected & $5.0 \pm 0.8$ & $5.0 \pm 0.7$ & $4.8 \pm 0.7$ & $5.0 \pm 0.7$ & 0.13 & $p<.336$ \\
\hline \multirow{5}{*}{$\begin{array}{l}\text { Cholesterol } \\
\text { [nmol/L] }\end{array}$} & Group A & $3.8 \pm 0.8$ & $3.8 \pm 0.7$ & $3.8 \pm 0.7$ & $3.8 \pm 0.6$ & 0 & \\
\hline & Group B & $3.6 \pm 0.6$ & $3.6 \pm 0.5$ & $3.6 \pm 0.5$ & $3.5 \pm 0.4$ & 0.19 & \\
\hline & Group C & $3.5 \pm 0.5$ & $3.5 \pm 0.5$ & $3.5 \pm 0.5$ & $3.3 \pm 0.9$ & 0.27 & \\
\hline & Total & $3.6 \pm 0.6$ & $3.6 \pm 0.6$ & $3.6 \pm 0.6$ & $3.5 \pm 0.7 \S$ & 0.15 & \\
\hline & Corrected & $3.6 \pm 0.6$ & $3.5 \pm 0.6$ & $3.4 \pm 0.5^{*+}$ & $3.4 \pm 0.7^{*+}$ & 0.30 & $p<.001$ \\
\hline \multirow{5}{*}{$\begin{array}{l}\mathrm{HDL} \\
{[\mathrm{nmol} / \mathrm{L}]}\end{array}$} & Group A & $1.44 \pm 0.3$ & $1.49 \pm 0.3$ & $1.48 \pm 0.4$ & $1.38 \pm 0.3^{\dagger \S}$ & 0.20 & \\
\hline & Group B & $1.38 \pm 0.2$ & $1.44 \pm 0.2$ & $1.42 \pm 0.3$ & $1.24 \pm 0.2^{x+\S}$ & 0.27 & \\
\hline & Group C & $1.26 \pm 0.2$ & $1.28 \pm 0.2$ & $1.28 \pm 0.2$ & $1.11 \pm 0.2^{x+\S}$ & 0.74 & \\
\hline & Total & $1.39 \pm 0.3$ & $1.42 \pm 0.3$ & $1.39 \pm 0.3$ & $1.27 \pm 0.3^{x+\S}$ & 0.36 & \\
\hline & Corrected & $1.38 \pm 0.3$ & $1.40 \pm 0.3$ & $1.32 \pm 0.3^{+}$ & $1.22 \pm 0.3^{x}+\S$ & 0.53 & $p<.001$ \\
\hline \multirow{5}{*}{$\begin{array}{l}\text { LDL } \\
{[\mathrm{nmol} / \mathrm{L}]}\end{array}$} & Group A & $2.02 \pm 0.6$ & $2.23 \pm 0.5^{*}$ & $2.20 \pm 0.5$ & $2.12 \pm 0.5$ & 0.20 & \\
\hline & Group B & $2.00 \pm 0.4$ & $2.13 \pm 0.4$ & $2.06 \pm 0.3$ & $2.00 \pm 0.4$ & 0 & \\
\hline & Group C & $2.05 \pm 0.4$ & $2.16 \pm 0.6$ & $2.02 \pm 0.4$ & $2.08 \pm 0.5$ & 0.06 & \\
\hline & Total & $2.04 \pm 0.5$ & $2.18 \pm 0.5^{*}$ & $2.08 \pm 0.4$ & $2.08 \pm 0.4^{+}$ & 0.08 & \\
\hline & Corrected & $2.04 \pm 0.5$ & $2.15 \pm 0.5^{*}$ & $2.00 \pm 0.4^{+}$ & $2.00 \pm 0.4^{\dagger}$ & 0.08 & $p<.014$ \\
\hline
\end{tabular}

", Significantly different from phase $T_{1} ;{ }^{+}$, significantly different from phase $T_{2}$; $\$$, significantly different from phase $T_{3},(p<.05)$. CK = creatine kinase; $A L T=$ alanine aminotransferase; $A S T=$ aspartame aminotransferase; $H D L=$ high-density lipoproteins; $L D L=$ low-density lipoproteins. 
Table 5

Cortisol, SHBG and electrolytes variables for the entire group of athletes and for each age group (mean $\pm S D$ ) at baseline, peak of the annual training cycle, competitions and at the beginning of the following preseason.

\begin{tabular}{|c|c|c|c|c|c|c|c|}
\hline & & Baseline & $\begin{array}{c}\text { Peak } \\
\text { training }\end{array}$ & Competitions & $\begin{array}{c}\text { Next } \\
\text { preseason }\end{array}$ & $\begin{array}{c}\text { Cohen's } \\
d\end{array}$ & $p$ \\
\hline Variable & Group & $\mathrm{T}_{1}$ & $\mathrm{~T}_{2}$ & $\mathrm{~T}_{3}$ & $\mathrm{~T}_{4}$ & $\mathrm{~T}_{1}-\mathrm{T}_{4}$ & \\
\hline \multirow{5}{*}{$\begin{array}{l}\text { Cortisol } \\
{[\mathrm{nmol} / \mathrm{L}]}\end{array}$} & Group A & $250.8 \pm 113$ & $215.4 \pm 75$ & $252.6 \pm 65$ & $160.6 \pm 72^{*}+\S$ & 0.94 & \\
\hline & Group B & $288.0 \pm 109$ & $232.5 \pm 80^{*}$ & $269.2 \pm 106$ & $151.3 \pm 76^{*}+\S$ & 1.45 & \\
\hline & Group C & $259.3 \pm 81$ & $264.5 \pm 125$ & $274.4 \pm 110$ & $238.2 \pm 82.2$ & 0.25 & \\
\hline & Total & $265.0 \pm 102$ & $235.7 \pm 95$ & $251.5 \pm 95$ & $181.8 \pm 85^{*}+\S$ & 0.88 & \\
\hline & Corrected & $265.0 \pm 102$ & $232.4 \pm 94$ & $239.2 \pm 91$ & $175.1 \pm 82^{x}+\S$ & 0.93 & $p<.001$ \\
\hline \multirow{5}{*}{$\begin{array}{l}\text { SHBG } \\
{[\mathrm{nmol} / \mathrm{L}]}\end{array}$} & Group A & $70.3 \pm 33.4$ & $135.4 \pm 67^{*}$ & $90.2 \pm 42.9^{* t}$ & $67.8 \pm 37.6^{+8}$ & 0.07 & \\
\hline & Group B & $91.6 \pm 64.4$ & $91.4 \pm 52$ & $58.0 \pm 32.3^{*+}$ & $41.8 \pm 20.2^{*} \S$ & 1.04 & \\
\hline & Group C & $67.8 \pm 39.2$ & $65.2 \pm 25$ & $35.8 \pm 16.3^{*+}$ & $35.3 \pm 29.8^{*}+\S$ & 0.93 & \\
\hline & Total & $76.0 \pm 45.8$ & $97.9 \pm 62^{*}$ & $62.1 \pm 40.4^{*+}$ & $48.7 \pm 32.6^{*}+\S$ & 0.68 & \\
\hline & Corrected & $76.0 \pm 45.8$ & $96.5 \pm 60.7^{*}$ & $59.0 \pm 38.4^{*+}$ & $46.9 \pm 31.3^{*}+\S$ & 0.16 & $p<.001$ \\
\hline \multirow{5}{*}{$\begin{array}{l}\text { Sodium } \\
{[\mathrm{nmol} / \mathrm{L}]}\end{array}$} & Group A & $138.9 \pm 1.6$ & $138.6 \pm 2.3$ & $137.9 \pm 1.2^{*}$ & $139.0 \pm 1.5^{*}$ & 0.06 & \\
\hline & Group B & $138.3 \pm 1.8$ & $137.7 \pm 1.9$ & $138.6 \pm 1.8^{+}$ & $139.1 \pm 1.7^{+}$ & 0.45 & \\
\hline & Group C & $138.9 \pm 1.5$ & $138.8 \pm 1.5$ & $139.6 \pm 1.9$ & $139.9 \pm 1.4^{*} \S$ & 0.68 & \\
\hline & Total & $138.7 \pm 1.6$ & $138.4 \pm 2.0$ & $138.8 \pm 1.8$ & $139.3 \pm 1.6$ & 0.37 & \\
\hline & Corrected & $138.7 \pm 1.6$ & $136.5 \pm 2.0^{*}$ & $132.0 \pm 1.7^{*+}$ & $134.1 \pm 1.5^{*}+\S$ & 0.28 & $p<.001$ \\
\hline \multirow{5}{*}{$\begin{array}{l}\text { Potassium } \\
{[\mathrm{nmol} / \mathrm{L}]}\end{array}$} & Group A & $4.5 \pm 0.4$ & $4.4 \pm 0.4$ & $4.4 \pm 0.4$ & $4.3 \pm 0.2^{*}$ & 0.35 & \\
\hline & Group B & $4.3 \pm 0.3$ & $4.3 \pm 0.3$ & $4.3 \pm 0.2$ & $4.3 \pm 0.2$ & 0 & \\
\hline & Group C & $4.2 \pm 0.2$ & $4.3 \pm 0.3$ & $4.4 \pm 0.3$ & $4.2 \pm 0.4$ & 0 & \\
\hline & Total & $4.3 \pm 0.3$ & $4.3 \pm 0.3$ & $4.4 \pm 0.3$ & $4.3 \pm 0.3$ & 0 & \\
\hline & Corrected & $4.3 \pm 0.3$ & $4.2 \pm 0.3$ & $4.2 \pm 0.3$ & $4.1 \pm 0.3^{*}+\S$ & 0.21 & $p<.001$ \\
\hline \multirow{5}{*}{$\begin{array}{l}\text { Chloride } \\
{[\mathrm{nmol} / \mathrm{L}]}\end{array}$} & Group A & $101.7 \pm 1.6$ & $101.8 \pm 1.7$ & $100.9 \pm 1.3^{*+}$ & $102.5 \pm 0.3^{*}+\S$ & 0.33 & \\
\hline & Group B & $101.6 \pm 1.3$ & $101.2 \pm 1.2$ & $101.2 \pm 2.0$ & $102.3 \pm 1.3^{*+}$ & 0.53 & \\
\hline & Group C & $101.2 \pm 1.7$ & $102.0 \pm 1.6^{*}$ & $102.3 \pm 1.9^{*}$ & $102.1 \pm 1.9$ & 0.49 & \\
\hline & Total & $101.5 \pm 1.5$ & $101.8 \pm 1.6$ & $101.6 \pm 1.8$ & $102.4 \pm 1.6^{*}+\S$ & 0.58 & \\
\hline & Corrected & $101.5 \pm 1.5$ & $100.4 \pm 1.6^{*}$ & $96.6 \pm 1.7^{*+}$ & $98.6 \pm 1.51^{*}+\S$ & 1.39 & $p<.001$ \\
\hline
\end{tabular}

", Significantly different from phase $\mathrm{T}_{1}{ }^{+}{ }^{+}$, significantly different from phase $\mathrm{T}_{2}$;

$\S$, significantly different from phase $T_{3},(p<.05)$. SHBG $=$ sex hormone binding globulin.

Moreover, studies exploring the effects of different training programs on various biochemical variables have often not considered the possible role of plasma volume shifts (Kargotish et al., 1998). Most of studies have not mentioned or accounted for plasma volume changes and few studies, although have included plasma volumes shifts, have not corrected the extracted data correspondingly.

Sports' training has been suggested to determine "sports anaemia" mainly caused by plasma volume expansion primarily due to 
endurance training. The plasma volume expansion has been shown to occur more rapidly and to a greater extent than the increase in red cell volume following endurance-training programmes (Weight et al., 2007). In two studies a plasma volume expansion was observed in young soccer athletes and older swimmers from baseline to the end of the examined period (Andelcovic et al., 2015; Santhiago et al., 2009). However, in our study, plasma volume was decreased in the whole group by $1.4 \%$ after 5 months of regular training $\left(\mathrm{T}_{2}\right)$ and by $4.9 \%$ in May $\left(\mathrm{T}_{3}\right)$.

Our study suggests that while some significant variations in haematological variables like Hct and MCV can be evident over the course of an annual training cycle in this age group, the effect size is rarely large, except for MCHC. In some individual sports, such as cycling, running and swimming, declines in $\mathrm{Hb}$ and Hct concentrations have been documented with intensive training and competition activities in adult athletes (Guglielmini et al., 1989; Pizza et al., 1997; Schumacher et al., 2002). However, relatively recent work (Diaz et al., 2010) suggested that some hematological values like the reticulocytes percentage (Ret\%) and haemoglobin $(\mathrm{Hb})$ were relatively stable over four consecutive seasons in elite triathletes implying that in adults variability should be limited. The observed fluctuations of haematological variables of our cohort are quite similar to what was observed in Serbian soccer players where their $\mathrm{Hb}$ concentrations remained unaltered, but the Hct concentration gradually increased up to $44 \pm 2$ [\%] at the end of the annual training cycle (Ostojic and Ahmetovic, 2009). Also, data from our study show that Arab adolescents present values similar to those observed in same age groups in other parts of the World (Yip et al., 1984) and it is likely that the observed significant increase in Hct, MCV and Ret-He is a reflection of growth and maturation rather than training-induced alterations.

The mean cell volume (MCV) is another variable that presented relatively lower values in our population as compared to similar age groups. Our athletes in the two younger groups (A and B) started the season with even lower $\mathrm{MCV}$, while the MCV for the older group $C$ was marginally above 80 [fl], which is the proposed threshold of microcytosis prevalence (Irwin and Kirchner, 2001). The whole process of erythropoiesis can and should be monitored adequately through the measurement of Ret $\%$, Ret-He and IRF. These variables were marginally affected (Cohen's $d \leq 0.2$ ) by yearly training in the entire sample of our tested population. Total iron and ferritin levels in this entire cohort were within normal clinical ranges and their changes over the year showed a small effect size. Previous research on team sports athletes has noted an increase in ferritin levels after the initial training phase (Andelcovic et al., 2014).

\section{White blood cells and subpopulations}

Our findings showed that there was a considerable reduction in leukocytes between baseline $\left(T_{1}\right)$ and the competitive period $\left(T_{3}\right)$, while neutrophils, lymphocytes, monocytes and eosinophils percentages had variations with trivial to small effect sizes. Concerning the impact of regular exercise on platelet levels, most researchers agree that different kinds of exercise could considerably increase the number of platelets. Such increases have been found in different sports events such as a marathon race, a middle distance run, in triathlon, in soccer and many other sports (Souglis and Travlos, 2015). However, in our cohort, platelets count decreased significantly over the year with a small effect size.

\section{Various biochemical markers}

Creatine kinase activity in serum is an indirect marker of muscle cell damage and is usually elevated following strenuous activity. It is also known that higher permeability of muscle cell membranes exists in some ethnic groups (Wong et al., 1983), and certain individuals like athletes with high percentages of fast twitch (type 11) muscle fibres also tend to have higher CK values than controls (Meyer and Meister, 2011). In a parallel pattern, our cohort of young athletes presented higher $\mathrm{CK}$ values compared to the general population; besides, these values remained above the proposed reference range for the complete annual training cycle. The specific population based analysis revealed an upper limit of 1,394 U/L. Despite the fact that some of those values might have been linked to activities performed by the athletes in the 48 hours preceding the screening, it is possible that this particular variable should be analysed in the context of individual variability in athletic populations rather than absolute values within clinical ranges as previously suggested. 
Our results of aminotransferases suggest that such variables are relatively stable in young Arab athletes. A gradual increase in creatinine concentration was observed during the annual training cycle with a moderate effect size. Literature has revealed higher creatinine values in athletes compared to the non-athletic population. The reasons for high creatinine concentrations included increased muscle mass leading to greater creatinine turnover than usual and creatine intake in athletes (Banfi, 2010). In a more recent study in young soccer players, values of serum creatinine exceeded the reference intervals for the general population over the observational period in line with our findings (Andelcovic et al., 2014). For our athletes, although they had gradually increased their creatinine concentrations during the annual training cycle with a moderate effect size, they never exceeded the upper limits of the clinical ranges reported for the general population.

Exercise and regular training have a significant impact on the lipids and lipoprotein levels of athletes. In fact, it has been previously documented that participation in sports has a positive effect on athletes compared to nonathletes with reference to the lipid status markers (Manna et al., 2010; Nikolaidis et al., 2003).

Exercise and regular training have also been reported to affect the neuroendocrine system. Cortisol has often been suggested as a useful biomarker to indicate overreaching or overtraining status, for its role in gluconeogenesis via the proteolytic pathway and the potential to represent a good marker of stress (Urhausen et al., 1995). Resting elevated cortisol levels generally reflect long-term training stress. Cortisol levels detected in our cohort marginally varied during the year but appeared remarkably reduced after the summer recovery period (moderate effect, Cohen's $d=0.93$ ) suggesting that the reduction in a training load typical of the summer break was effective in reducing resting cortisol levels. This trend was observed in corresponding to the SHBG concentration possibly influenced also by growth and maturation of our youth cohort. SHBG and albumin are the main proteins that are bound to and transfer testosterone.

Additionally, serum electrolytes when corrected for plasma volume changes, exposed significant decreases over the course of the annual training cycle. These reductions were lower than the proposed reference ranges for sodium and chloride during the competitive period in May, and these concentrations remained below the lower proposed threshold until the start of the following training cycle $\left(\mathrm{T}_{4}\right)$. However, the biological meaningfulness of such changes is unclear and could be associated with the hydration status of the participants at the time of blood collection.

Based on the results obtained in this study, we may summarize that training combined with growth and maturation in adolescent athletes can determine important changes in various haematological and biochemical markers related to health and performance. The knowledge of typical variations, clinical ranges and magnitude of the changes is of relevance in order to enhance training feedback as well as establish normative references for young athletic populations. Also, the results of our study indicate that while overall changes in an adolescent population are small in selected variables over a twelve month period, more considerable variations may be evident in athletes after plasma volume shifts are accounted for.

\section{Acknowledgements}

The Authors would like to thank the talent ID team of Aspire Academy and Mr. Andrew Douglas for the collection of anthropometric data.

\section{References}

Andelcovic M, Baralic I, Dordevic B, Stevuljevic JK, Radivojevic N, Dikic N, Skodric R, Stojkovic M. Hematological and Biochemical Parameters in Elite Soccer Players during a Competitive Half Season. J Med Biochem, 2015; 33: 1-7

Banfi G. Serum creatinine concentrations in athletes: are they normal? Brazilian Journal of Biomotricity, 2010; 4(3): 157-164 
Demorest R, Landry G. Training issues in elite young athletes. Current Sports Medicine Reports, 2004; 3(3): 167172

Diaz V, Lombardi G, Ricci C, Jacobs RA, Montalvo Z, Lundby C, Banfi G. Reticulocyte and haemoglobin profiles in elite triathletes over four consecutive seasons. Int Jnl Lab Hem, 2011; 33: 638-644

Dill DB, Costill DL. Calculation of percentage changes in volume of blood, plasma, and red cells in dehydration. J Appl Physiol, 1974; 37(2): 247-248

Eliakim A, Nemet D. The endocrine response to exercise and training in young athletes. Pediatric Exercise Science, 2013; 25(4): 605-615

Fallon KE. Utility of hematological and iron-related screening in elite athletes. Clinical Journal of Sport Medicine, 2004; 14(3): 145-152

Guglielmini C, Casoni I, Patracchini M, Manfredini F, Grazzi G, Ferrari M, Conconi F. Reduction of Hb levels during the racing season in non-sideropenic professional cyclists. Int J Sports Med, 1989; 10: 352-356

Hord JD. Anemia and coagulation disorders in adolescents. Adolesc Med, 1999; 10(3): 359-367

Joksimovic A, Stankovic D, Ilic D, Joksimovic I, Jerkan M. Hematological Profile of Serbian Youth National Soccer Teams. Journal of Human Kinetics, 2009; 22: 51-59

Irwin JJ, Kirchner JT. Anemia in children. Am Fam Physician, 2001; 64(8): 1379-86

Kargotich S, Goodman C, Keast D, Morton AR. The influence of exercise-induced plasma volume changes on the interpretation of biochemical parameters used for monitoring exercise, training and sport. Sports Med, 1998; 26(2): 101-117

Koehler K, Braun H, Achtzehn S, Hilderbrand U, Predel HG, Mester J, Schanzer W. Iron status in elite young athletes: Gender-dependent influences of diet and exercise. European Journal of Applied Physiology, 2012; 112(2): 513-523

Manna I, Khanna GL, Dhara PC. Effect of Training on Physiological and Biochemical Variables of Soccer Players of Different Age Groups. Asian Journal of Sports Medicine, 2010; 1(1): 5-22

Mirwald RL, Baxter-Jones ADG, Bailey DA, Beunen GP. An assessment of maturity from anthropometric measurements. Medicine and Science in Sports and Exercise, 2002; 34(4): 689-694

Meyer T, Meister S. Routine blood parameters in elite soccer players. Int J Sports Med, 2011; 32: 875-881

Nikolaidis MG, Protosygellou MD, Petridou A, Tsalis G, Tsigilis N, Mougios V. Hematologic and biochemical profile of juvenile and adult athletes of both sexes: Implications for clinical evaluation. International Journal of Sports Medicine, 2003; 24(7): 506-511

Ostojic SM, Ahmetovic Z. Indicator of iron status in elite soccer players during the sports season. Int J Lab Hematol, 2009; 31(4): 447-452

Pizza KX, Flynn MG, Boone JB, Rodriguez-Zayas JR, Andres FF. Serum haptoglobin and ferritin during a competitive running and swimming season. Int J Sports Med, 1997; 18(4): 233-237

Rogol A. Growth at puberty. Journal of adolescent health, 1994; 31: 192-200

Santhiago V, DaSilva ASR, Paroti M, Gobbato C. Responses of hematological parameters and aerobic performance of elite men and women swimmers during a 14- week training program. Journal of Strength and Conditioning Research, 2009; 23(4): 1097-1105

Schumacher YO, Schmid A, Grathwohl D, Bultermann D, Berg A. Hematological indices and iron status in athletes of various sports and performances. Medicine and Science in Sports and Exercise, 2002; 34(5): 869-875

Souglis A, Travlos A. The influence of competitive activity on selected biochemical and haematological parameters of amateur soccer athletes. Journal of Physical Education and Sport, 2015; 15(1): 24-31

Urhausen A, Gabriel H, Kindermann W. Blood Hormones as Markers of Training Stress and Overtraining. Sports medicine, 1995; 20(4): 251-276 
Yip R, Johnson C, Dallman PR. Prevalence and causes of anemia in the United States 1976 to 1980. Am J Clin Nutr, 1974; 39(3): 437-445

Weight LM, Klein M, Noakes TD, Jacobs P. "Sports anemia": a real or apparent phenomenon in endurancetrained athletes? Int. J. Sports Med, 1992; 13: 344-347

Winsley R, Matos N. Overtraining and Elite Young Athletes. Med Sport Sci, 2011; 56: 97-105

Wong ET, Cobb C, Umehara MK, Wolf G, Haywood LJ, Greenberg T, Shaw ST. Heterogeneity of serum creatine kinase activity among racial and gender groups of the population. Am J Clin Pathol, 1983; 79: $582-586$

\section{Corresponding author:}

\section{Dr Evdokia Varamenti}

c/o Aspire Academy, Department of Sports Science, PO Box 22287, Doha (Qatar),

E-mail: evdokia.varamenti@aspire.qa 